\title{
The effect of a baffle on the heat transfer in underground auxiliary ventilation systems
}

\author{
S. M. Aminossadati ${ }^{1}$ \& B. Ghasemi ${ }^{2}$ \\ ${ }^{1}$ CRCMining, School of Engineering, The University of Queensland, \\ Australia \\ ${ }^{2}$ Engineering Faculty, Shahrekord University, Iran
}

\begin{abstract}
This paper presents a computational fluid dynamics simulation to examine the effects of the baffle on the air flow field and the heat transfer rate in a space located beneath a ventilation channel. The computational model consists of a two-dimensional channel equipped with a thin baffle hanging from the top wall and a cavity located underneath simulating the underground space. Air flows trough the channel at a uniform velocity, $\mathrm{u}_{\mathrm{c}}$, and temperature, $\mathrm{T}_{\mathrm{c}}$. The cavity base is assumed to be heated at a constant temperature, $T_{h}$, while the vertical walls of the cavity are well-insulated. The top wall of the channel is maintained at a constant temperature, $T_{c}$. The continuity, momentum and energy equations are solved numerically using the control-volume approach to examine a combination of natural and forced convection flows. The results show that even though the existence of the baffle improves the cavity ventilation performance at low Richardson numbers, it has a negative impact at high Richardson numbers. Keywords: heat transfer, forced convection, underground ventilation, baffle.
\end{abstract}

\section{Introduction}

Nowadays, underground spaces are being utilised for a wide variety of purposes such as sport halls, power stations, waste repositories, mining operations and underground cities. The ventilation system is a key factor in the design of such spaces. Moreover, as the underground operations are continuing to develop to greater depths, the associated issues with the ventilation systems such as the heat load and airborne contaminants are becoming more important. 
Convection heat transfer in channels with open cavities has been commonly considered in the thermal design of underground spaces. A review of the literature indicates numerous studies on the flow field and heat transfer analysis of channels with open cavities Channels with open top upright cavities have been used by some researchers to simulate the heat transfer mechanism for either pure natural convection [1-5] or mixed convection [6-8]. Recently, Manca et al. [9] presented a numerical study on mixed convection in an open cavity with a heated wall bounded by horizontally insulated plate. They considered three basic heating modes for the open cavity: Assisting flow mode, opposing flow mode and heating from below mode. It was demonstrated that opposing flow mode had the highest thermal performance in terms of both maximum temperature and average Nusselt number. Brown and Lai [10] considered a horizontal channel with an open cavity and studied the combined heat and mass transfer numerically. In their study, only the bottom wall of the cavity was heated at a constant temperature and the other walls of the cavity were insulated. Correlations were obtained for combined heat and mass transfer which covered the entire convection regime from natural, mixed, to forced convection. Leong et al. [11] studied the mixed convection for the same geometry used by [10], showing that the Reynolds number and Grashof number control the flow pattern and the occurrence of recirculating cells while the cavity aspect ratio has a significant influence on the orientation of these cells. The transition to the mixed convection regime was a function of the relative magnitude of Grashof and Reynolds numbers. They argued that in the mixed convection regime, the heat transfer was reduced and the flow might have become unstable. Manca et al. [12] presented an experimental investigation on the mixed convection in a channel with open cavity having a heated wall on the inflow side. The geometry of the problem was similar to the one used by [9]. The results showed that the maximum dimensional temperature rise values decreased as the Reynolds and Richardson numbers decreased. For $\mathrm{Re}=1000$, two nearly distinct fluid motions were observed: a parallel forced flow in the channel and a recirculating flow inside the cavity. For $\mathrm{Re}=100$, the effect of a stronger buoyancy determined a penetration of thermal plume from the heated plate wall into the upper channel.

Auxiliary ventilation is often used in underground spaces to manage dust, gas and heat. Critical factors to auxiliary ventilation include the condition of supply air and the generation of dust, gas and heat in the underground space. The capacity of auxiliary ventilation systems is typically limited by the characteristics of the ducting system. A conductor baffle is often used in the ventilation channels to direct a portion of the ventilation air into a designated underground space. As such, the objective of this research is to study the effects of a baffle on mixed convection heat transfer in a two-dimensional horizontal channel with an open cavity.

\section{Problem definition}

The geometry considered here is a two-dimensional horizontal channel with an open cavity located at the bottom of the channel. Fig. 1 shows a schematic 
diagram of the channel. The channel is equipped with a thin baffle hanging from the top wall. Air is introduced into the channel at a uniform velocity, $u_{c}$, and temperature, $T_{c}$. The base of the cavity is at a high temperature, $T_{h}$, while the vertical walls of the cavity are insulated. The top wall of the channel is maintained at a low temperature, $T_{c}$, and the bottom wall is thermally insulated. The height of the inflow and outflow openings is equal to the depth of the cavity. This model can be used in the study of the effects of the baffle on the channel thermal performance, at various Richardson numbers.

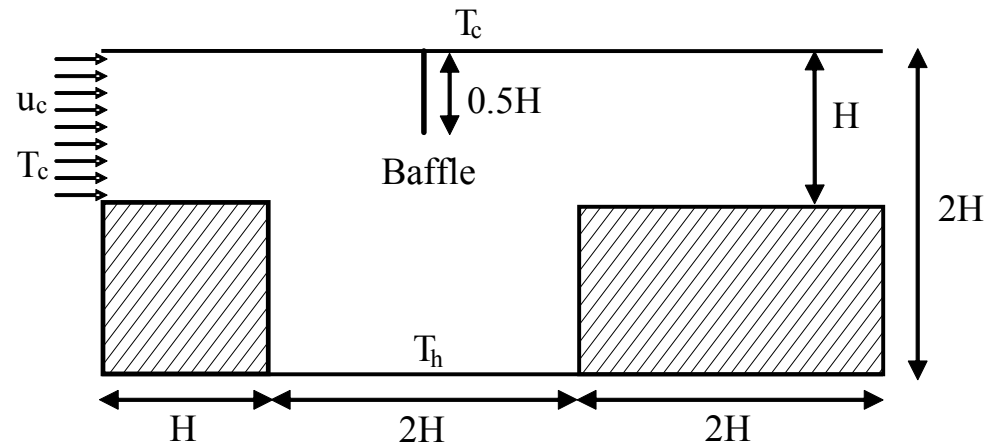

Figure 1: A schematic diagram of the model geometry.

\section{Governing equations}

By assuming, laminar, incompressible and two-dimensional convection fluid flow with constant properties except for the fluid density in buoyancy forces, the non-dimensional continuity, momentum and energy equations can be written as follows considering Boussinesq approximation:

$$
\begin{aligned}
& \frac{\partial \mathrm{U}}{\partial \mathrm{X}}+\frac{\partial \mathrm{V}}{\partial \mathrm{Y}}=0 \\
& \frac{\partial U}{\partial \tau}+U \frac{\partial U}{\partial X}+V \frac{\partial U}{\partial Y}=-\frac{\partial P}{\partial X}+\frac{1}{\operatorname{Re}}\left(\frac{\partial^{2} U}{\partial X^{2}}+\frac{\partial^{2} U}{\partial Y^{2}}\right) \\
& \frac{\partial V}{\partial \tau}+U \frac{\partial V}{\partial X}+V \frac{\partial V}{\partial Y}=-\frac{\partial P}{\partial Y}+\frac{1}{\operatorname{Re}}\left(\frac{\partial^{2} V}{\partial X^{2}}+\frac{\partial^{2} V}{\partial Y^{2}}\right)+\frac{G r}{\operatorname{Re}^{2}} \theta \\
& \frac{\partial \theta}{\partial \tau}+U \frac{\partial \theta}{\partial X}+V \frac{\partial \theta}{\partial Y}=\frac{1}{\operatorname{PrRe}}\left(\frac{\partial^{2} \theta}{\partial X^{2}}+\frac{\partial^{2} \theta}{\partial Y^{2}}\right)
\end{aligned}
$$

In these equations, the steady-state solutions can be obtained by setting the time dependence terms to zero. Based on the Boussinesq approximation, density is assumed to be constant in all the terms except for the buoyancy term. In the 
above non-dimensional equations, $\tau$ is the time in a dimensionless form $\left(\tau=u_{c} t / H\right)$, all the lengths are normalised by the depth of the cavity $(X=x / H, Y=y / H)$. Velocities are normalised by the inlet velocity $\left(U=u / u_{c}, V=v / u_{c}\right)$. The pressure is normalised as $P=\bar{p} / \rho u_{c}{ }^{2}$, where $\overline{\mathrm{p}}$ is the modified pressure $\left(p=p+\rho_{C} g y\right)$. The non-dimensional form of temperature is $\theta=\left(T-T_{c}\right) /\left(T_{h}-T_{c}\right)$. Fully-developed conditions are considered at the exit section of the channel.

Hydrodynamic Boundary Conditions: No slip boundary conditions $(U=V=0)$ on all the walls, $V=0, U=1$ at the entry section and $V=0, \partial U / \partial X=0$ at the exit section of the enclosure.

Thermal Boundary Conditions: $\theta=0$ at the entry section and for the top wall of the channel. $\theta=0$ for the base of the cavity. Adiabatic conditions $(\partial \theta / \partial X=0$ or $\partial \theta / \partial Y=0)$ are assumed for all other walls and at the exit section of the enclosure. For the unsteady analysis, $U=0, V=0$, and $\theta=0$ are considered as the initial conditions.

Grashof number is assumed to be constant $G r=10^{6}$; however, Richardson number, $\mathrm{Ri}=\mathrm{Gr} / \mathrm{Re}^{2}$, is varied in the range $0.01 \leq R i \leq 100$. Nusselt number, $N u$, is a measure of convective heat transfer coefficient at the bottom of the cavity. The local Nusselt number is defined as $N u=-(\partial \theta / \partial Y)_{Y=0}$ The average Nusselt number, $N u_{m}$, is obtained by integrating the Nusselt number over the bottom wall of the cavity.

\section{Numerical method}

The system of governing equations (1-4) with the above-mentioned boundary conditions is solved through a control volume formulation of the finite difference method. The well known SIMPLE algorithm is used to handle the pressurevelocity coupling. The convective fluxes across the surfaces of the control volume are determined by the power law discretisation scheme. A program code in Fortran is developed to follow the algorithm. In order to select the appropriate grid, a systematic grid independence study is carried out and the effect of grid refinement on the flow parameters is examined. According to the results of the grid independency study and for the sake of computation time, a equidistant grid of $110 \times 40$ is selected for the analysis.

\section{Results}

As the first step, the validation of the computer code for the mixed convection in the open cavity was assessed. This was done by running the present code with the conditions used by Manca et al. [9]. They considered an open cavity in a channel with a uniform heat flux on the vertical wall. Fig. 2 shows the variation of the minimum stream function with Richardson number. As can be seen from this figure, there is a good agreement between the two studies. 


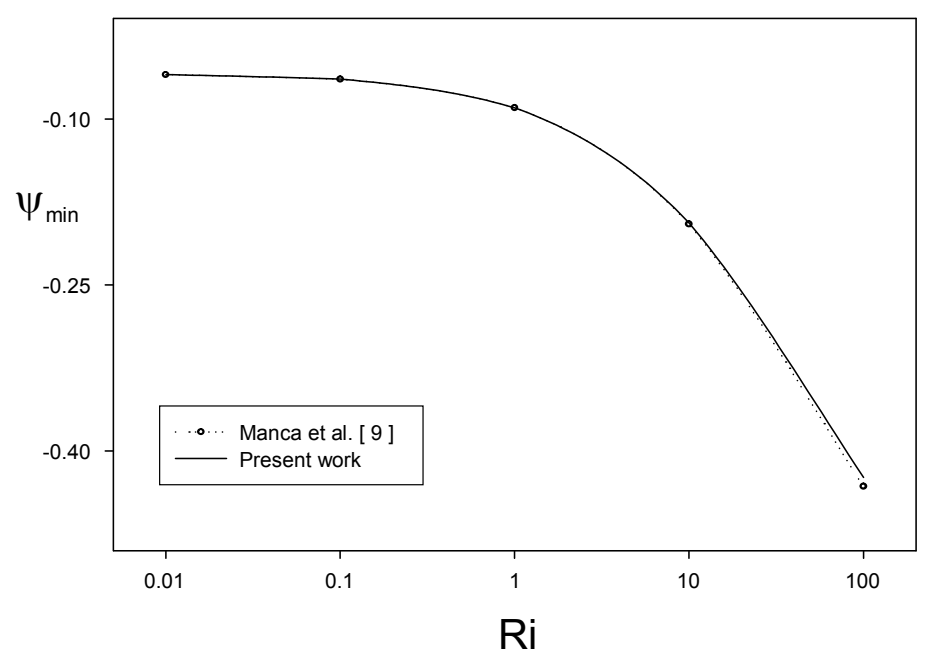

Figure 2: A comparison between the present work and Manca et al. [9].

The second step was to study the effects of baffle on the flow and temperature fields and the heat transfer rate. To accomplish this study, the following values are used in the analysis. $\operatorname{Pr}=0.71, G r=10^{6}$, and $0.01 \leq R i \leq 100$. The flow and isotherms are shown in Fig. 3. Streamlines and isotherms are presented on the left and right hand sides of the figure, respectively.

Plots presented in Fig. 3(a) correspond to three different values of the mixed convection parameter $\mathrm{Ri}=0.1,1$, and 10 at a channel with no baffle, whereas the plots in Fig. 3(b) are for a channel in which a baffle is installed. Fig. 3(a), shows that at $\mathrm{Ri}=0.1$, the airflow does not have any tendency to enter the cavity and as a result, an insignificant circulation flow is evident in the cavity. As the Richardson number increases, stronger buoyancy cells appear in the cavity and begin to cover the whole cavity. It can be said that, an increase in Richardson number $\left(\mathrm{Re}=\mathrm{Gr} / \mathrm{Re}^{2}\right)$ is associated with a decrease in Reynolds number since Grashof number is assumed constant. Consequently, the externally induced airflow in the channel become less controlling and that associates with the onset of natural flow circulations. In the next scenario, as seen in Fig. 3(b), the baffle directs some portion of the external flow into the cavity and therefore circulation cells are generated in the entire cavity at low Richardson number of $\mathrm{Ri}=0.1$, The isotherms also show an improvement in the penetration of the external flow into the cavity when the baffle is used.

Fig. 4 shows the vertical velocity component of the airflow at the horizontal midsection of the cavity, $\mathrm{Y}=0.5$, The plots for both channels with and without baffle are presented in this figure. For small Richardson numbers, the peak of the velocities in the channel without baffle is almost flat showing only a slight buoyancy effect. In the cavity of the channel with baffle, the recirculation zones appear at low Richardson number (see Fig. 3(b)) and sharp changes in the slope of the velocity profiles observed near the vertical walls of cavity. As the 

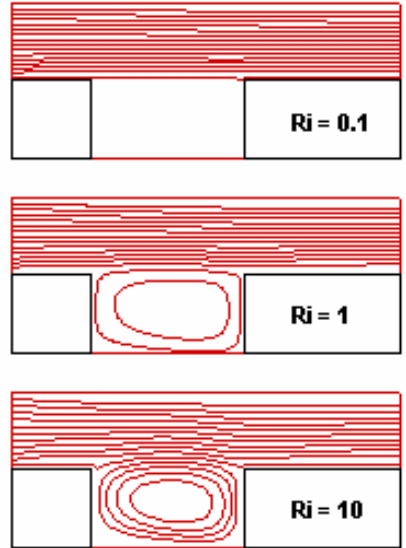

(a)
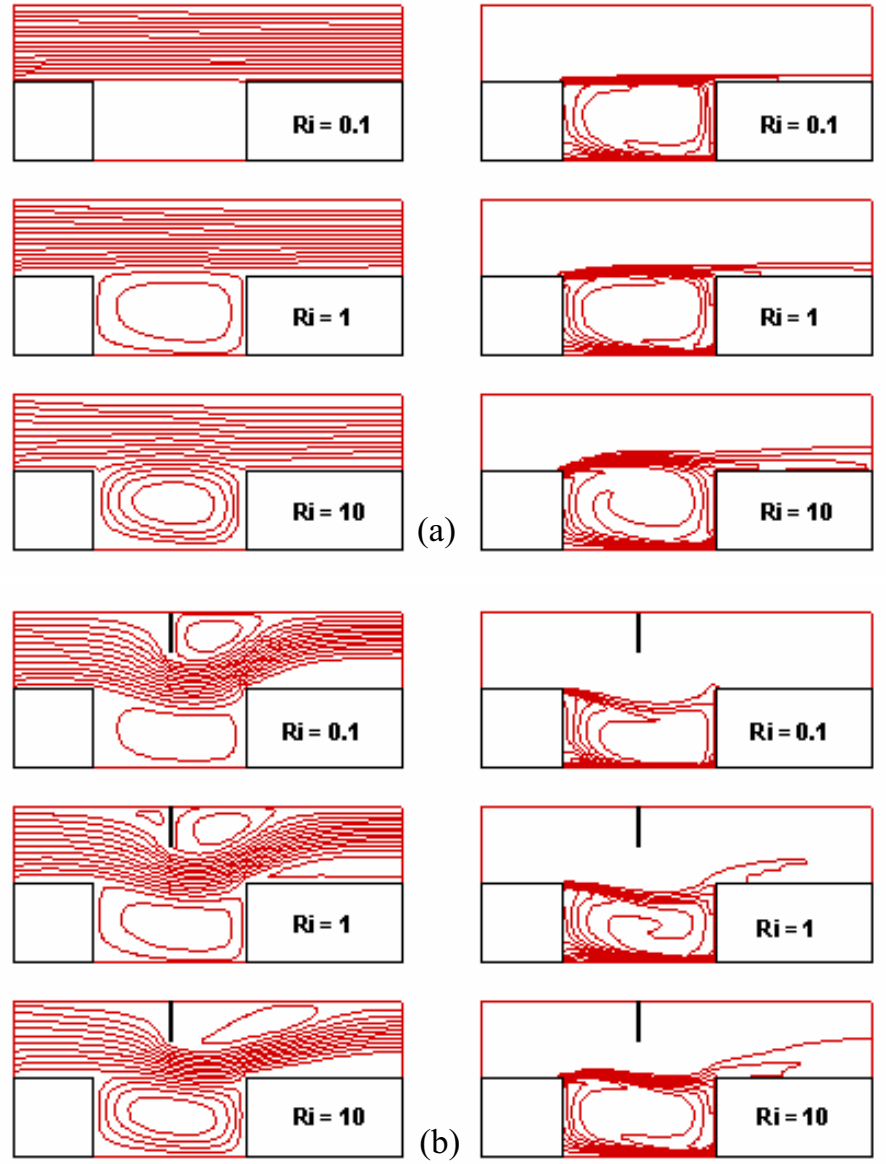

(b)

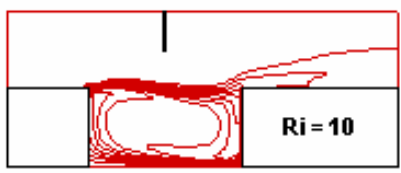

Figure 3: Streamlines (left) and isotherms (right) at various Richardson numbers (a) channel without baffle, (b) channel with baffle.

Richardson number increases, the recirculation zones become stronger in both cavities and the peak of the velocity profiles moves upwards. In this case, only a slight difference is evident between two channel profiles.

The variation of average temperature of the entire cavity is presented in Fig. 5. The results show that for the channel with no baffle the average temperature does not change significantly with low Richardson numbers. In addition, it can be seen that at Richardson number of $\mathrm{Ri}=0.01$, the average temperature for the channel with baffle is much lower than that for the channel without baffle and that is because of the baffle directs some portion of the dominated external air flow with a relatively lower temperature into the cavity. As Richardson number increases, the average temperature increases because the buoyant flow becomes stronger. An interesting point is that at $\mathrm{Ri}=100$, the average temperature for the channel with baffle is higher than that for the channel without baffle. This will be explained in the subsequent sections. 


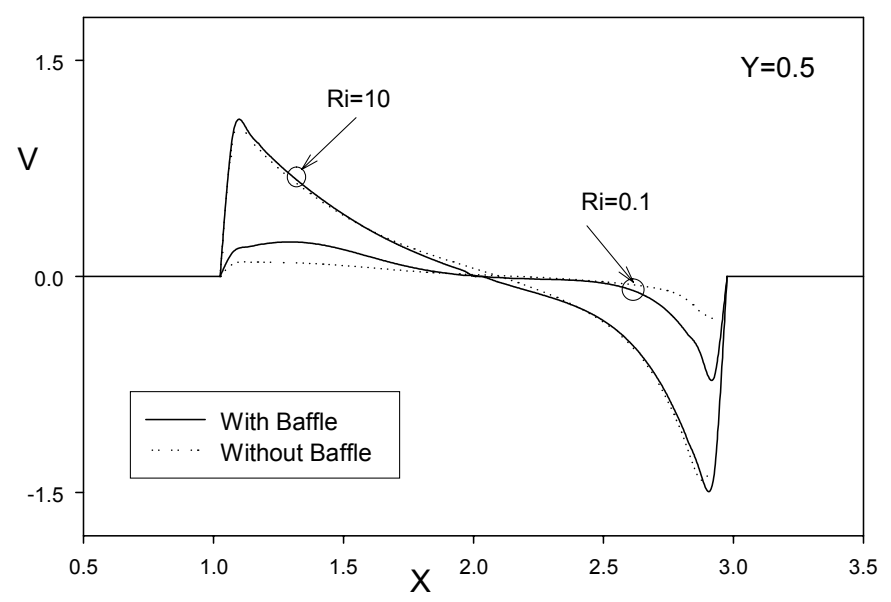

Figure 4: Vertical component of velocity at midsection of the cavity.

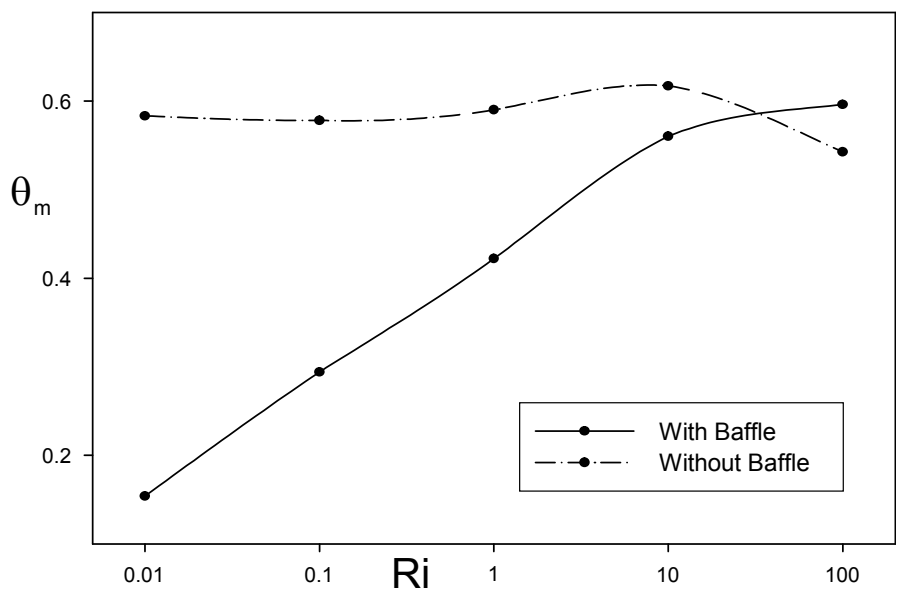

Figure 5: Average air temperature in the cavity.

Heat transfer rate from the cavity in terms of the variation of average Nusselt number for the two channels with and without a baffle is presented in Fig. 6. As expected, at low and medium Richardson numbers, the cavity of the channel with baffle has a better thermal performance.

A better comparison of the heat transfer performance of the two different channels is presented in Fig. 7. Here, the variations of the average fluid temperature ratio, $\theta_{m B} / \theta_{m}$, and the average Nusselt number ratio, $N u_{m B} / N u_{m}$ are shown. The results of the average Nusselt number ratio shows that at $\mathrm{Ri}=0.01$, the heat transfer rate for the channel with baffle is approximately three times higher than that for the other channel. 


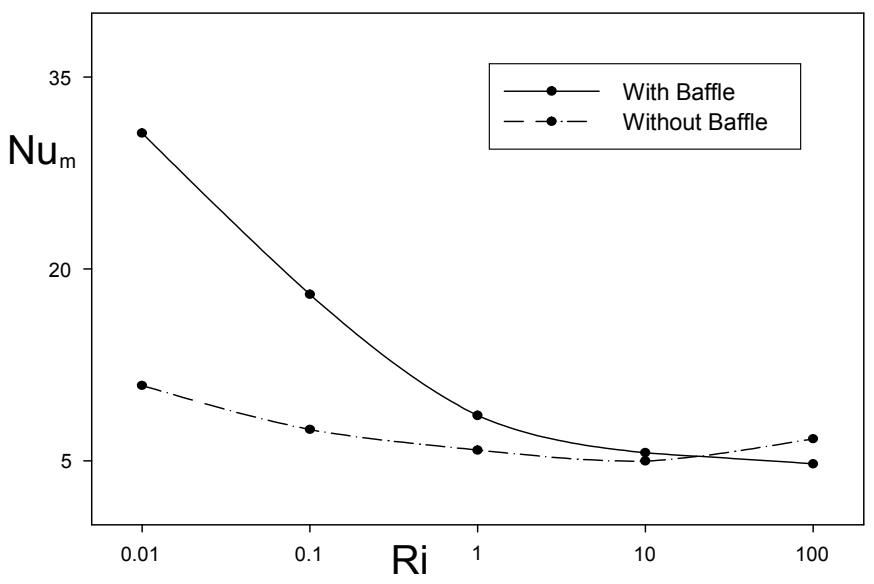

Figure 6: Average Nusselt number of the cavity.

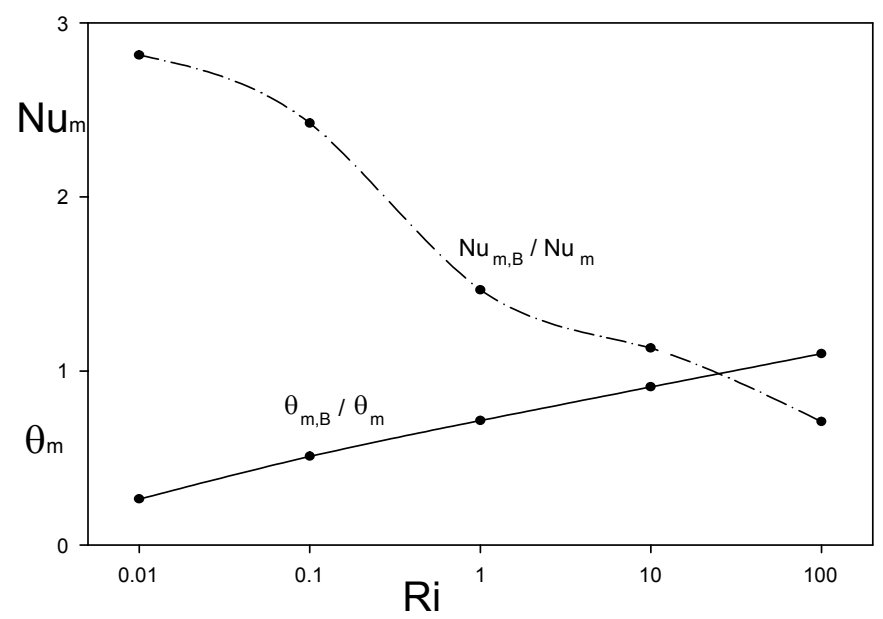

Figure 7: Average air temperatures and average Nusselt numbers ratios.

The results obtained at $\mathrm{Ri}=100$ in terms of cavity average temperature and the average Nusselt number can be related to the fluctuating results of the flow field at this Richardson number. In fact, no steady solution was found due to the instability in the physical system for this situation. Other researchers also found the fluctuating results in closed and open cavities and discussed that the Hopf bifurcations lead to oscillating flows $[13,14]$. In this work, the examination of heat transfer at $\mathrm{Ri}=100$ is based on solving the unsteady equations using dimensionless time step, $\Delta \tau=0.1$. Fig. 8 shows the time variation of the average cavity air temperature, $\theta_{m}$ for the two channels. It can be seen that the 
channel with baffle has a lower frequency and higher amplitude fluctuations. The steady state values of $\theta_{m}$ and $N u_{m}$, presented in Figs 5, 6, and 7, are the mean time value in the asymptotic periodic zone.

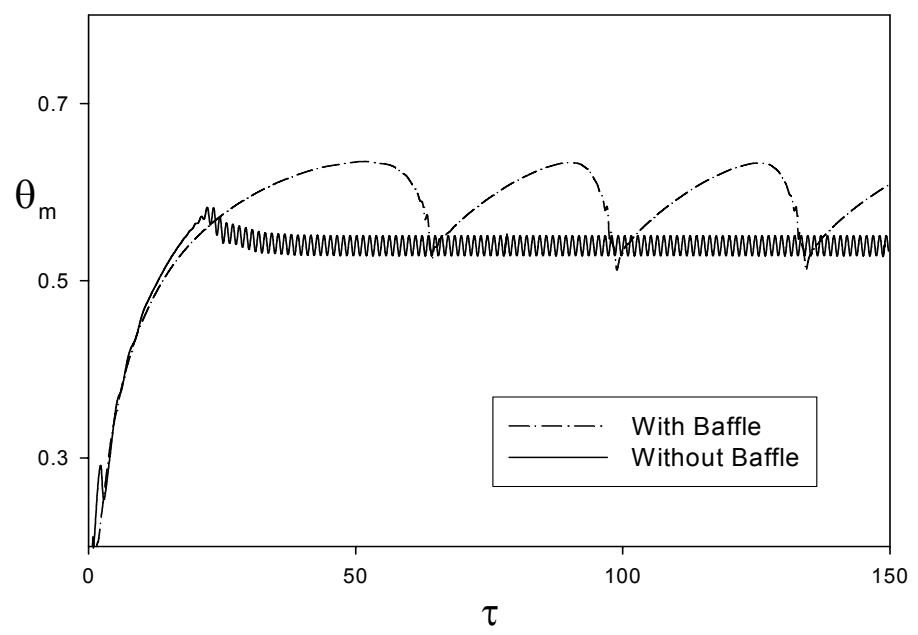

Figure 8: $\quad$ Time variation of average air temperature in cavity at $\mathrm{Ri}=100$.

\section{Conclusions}

In this study, the results of a numerical modelling of mixed convection in an open cavity beneath of a two-dimensional channel with and without baffle are presented. The results of the average temperatures and average heat transfer have been performed for a series of Richardson numbers. The results show that, for low and medium Richardson numbers, the baffle has a key roll in the ventilation of the cavity; however, at a high Richardson number of $\mathrm{Ri}=100$, the baffle has a negative effect on the cooling performance of the cavity.

\section{References}

[1] M. Hasnaoui, E. Bilgen, P. Vasseur, Natural convection above an array of open cavities heated from below, Numerical Heat Transfer, Part A, 18 pp. 463-482, 1990.

[2] G.F. Jones, J. Cai, Analysis of a transient asymmetrically heated/cooled open thermosyphon, Journal of Heat Transfer, 115 pp. 621-630,1993.

[3] R.A. Showole, J.D. Tarasuk, Experimental and numerical studies of natural convection with flow separation in upward-facing inclined open cavities, Journal of Heat Transfer, 115(3), pp. 592-605, 1993. 
[4] S.W. Chang, S. Chiou, L. Su, T. Yang, Free connective heat transfer in tilted longitudinal open cavity, Heat Transfer Engineering, 26(10) pp.4664, 2005.

[5] M. El Alami, M. Najam, E. Semma, A. Oubarra, F. Penot, Electronic components cooling by natural convection in horizontal channel with slots, Energy Conversion and Management, 46(17), pp. 2762-2772, 2005.

[6] K. Vafai, C.P. Desai, S.V. Iyer, M.P. Dyko, Buoyancy induced convection in a narrow open-ended annulus, ASME Journal of Heat Transfer, 119(3), pp. 483-494, 1997.

[7] T. Fusegi, Numerical study of convective heat transfer from periodic open cavities in a channel with oscillatory throughflow, International Journal of Heat and Fluid Flow, 18(4), pp. 376-383, 1997.

[8] K. Khanafer, K. Vafai, Buoyancy-driven flow and heat transfer in openended enclosures: elimination of the extended boundaries, International Journal of Heat and Mass Transfer, 43(22), pp. 4087-4100, 2000.

[9] O. Manca, S. Nardini, K. Khanafer, K. Vafai, Effect of heated wall position on mixed convection in a channel with an open cavity, Journal of Numerical Heat Transfer, 43(3), pp. 259-282, 2003.

[10] N.M. Brown, F.C. Lai, Correlations for combined heat and mass transfer from an open cavity in a horizontal channel, International Communications in Heat and Mass Transfer, 32(8), pp. 1000-1008, 2005.

[11] J.C. Leong, N.M. Brown, F.C. Lai, Mixed convection from an open cavity in a horizontal channel, International Communications in Heat and Mass Transfer, 32(5), pp. 583-592, 2005.

[12] O. Manca, S. Nardini, K. Vafai, Experimental investigation of mixed convection in a channel with an open cavity, Experimental Heat Transfer, 19(1), pp. 53-68, 2006.

[13] E. Papanicolaou, Y. Jaluria, Transition to a periodic regime in mixed convection in a square cavity, Journal of Fluid Mechanics, 239 pp. 489$509,1992$.

[14] M.C. D’Orazio, C. Cianfrini, M. Corcione, Rayleigh-Bénard convection in tall rectangular enclosures, International Journal of Thermal Sciences, 43(2), pp. 135-144, 2004. 